\title{
Autonomous system to control a mobile robot
}

\author{
Ayman Abu Baker ${ }^{1}$, Yazeed Yasin Ghadi ${ }^{2}$ \\ ${ }^{1}$ Department of Electrical Engineering, Applied Science Private University, Jordan \\ ${ }^{2}$ Department of Electrical Engineering, Al Ain University of Science and Technology, UAE
}

\begin{tabular}{|c|c|}
\hline Article Info & ABSTRACT \\
\hline Article history: & \multirow{9}{*}{$\begin{array}{l}\text { This paper presents an ongoing effort to control a mobile robot in unstructured } \\
\text { environment. Obstacle avoidance is an important task in the field of robotics, } \\
\text { since the goal of autonomous robot is to reach the destination without } \\
\text { collision. Several algorithms have been proposed for obstacle avoidance, } \\
\text { having drawbacks and benefits. In this paper, the fuzzy controller is used to } \\
\text { tackle the problem of mobile robot autonomous navigation in unstructured } \\
\text { environment. The objective is to make the robot move along a collision free } \\
\text { trajectory until it reaches its target. The proposed approach uses the fuzzified, } \\
\text { adaptive inference engine and defuzzification engine. Also number of linguistic } \\
\text { labels is optimized for the input of the mobile robot in order to reduce } \\
\text { computational time for real-time applications. The proposed fuzzy controller } \\
\text { is evaluated subjectively and objectively with other approaches and also } \\
\text { the processing time is taken in consideration. }\end{array}$} \\
\hline Received Dec 1, 2019 & \\
\hline Revised Feb 21, 2020 & \\
\hline Accepted Mar 23, 2020 & \\
\hline Keywords: & \\
\hline Fuzzy logic & \\
\hline Mobile robot & \\
\hline Navigation sy & \\
\hline Unstructured environment & \\
\hline
\end{tabular}

This is an open access article under the CC BY-SA license.

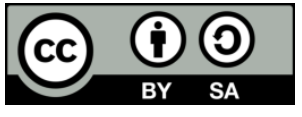

\section{Corresponding Author:}

Ayman Abu Baker,

Department of Electrical Engineering,

Applied Science Private University,

166-11931, Shafa Badran, Amman, Jordan.

Email: a_abubaker@asu.edu.jo

\section{INTRODUCTION}

Among all the soft-computing methods suggested for mobile robot reactive navigation, fuzzy logic systems have been found to be the most attractive. They are tolerant to noise and error in the sense of information coming from the sensory system, and most importantly they are factual reflection of the behavior of human expertise. In general, there are two approaches to the application of fuzzy logic in mobile robot navigation, namely, behavior-based approach [1-3] and classical fuzzy rule-base approach [4-6]. The design of fuzzy logic rules is often reliant on heuristic experience and it lacks systematic methodology. Therefore this rules might not be correct and consistent, do not possess complete domain knowledge, and/or could have a proportion of redundant rules. Furthermore, when a better precision is needed the number of input variables and their fuzzy values need to be increased, for example, when using four input variables each mapped by seven fuzzy values besides 2401 if-then rules maybe required to define the rule-base of the inference system. Such huge expansion in a multi-dimensional fuzzy rule-based system adds further ad hoc to the design of the system [7].

Several successful reactive navigation approaches based on neural networks have been suggested in the literature [8-10]. In spite of various suggested network topologies and learning methods, neural reactive navigators still perceive their knowledge and skills from demonstrating actions. Therefore, they suffer from a very slow convergence, lack of generalization due to limited patterns to represent complicated environments, and finally information encapsulated within the network can not be interpreted into physical knowledge [11]. Consequently, the utilization of neural networks in reactive mobile robot navigation is limited when compared to fuzzy logic. However, the role of neural networks has been found to be very useful and effective 
when integrated with fuzzy systems [12]. The birth of this integration between these two soft-computing paradigms is the neuro-fuzzy systems. Neuro-fuzzy systems provide an urgent synergy that can be found between the two paradigms, especially the capability to mimic human experts as in fuzzy logic, and learning from previous experience capability as in neural networks.

In general, neuro-fuzzy systems can be classified into three categories, neurally adaptive fuzzy inference system, neurally performed FIS, and combinatorial, or hybrid, neuro-fuzzy systems. The neurally adaptive fuzzy inference system is the most widely used neuro-fuzzy systems, and they are designed to combine the learning capabilities of neural networks and reasoning properties of fuzzy logic [13]. In this paper, a new approach is proposed to design a simple fuzzy navigation system. The proposed system has apparent advantage in structures that simplify and reduce the processing time and improve the performance. This archived by using a significant number of fuzzy rules. With such a technique, the required time needed to infer the decision for the robot movement is greatly reduced.

\section{LITERATURE REVIEW}

Previous authors have applied various types of techniques to control the mobile robot using different intelligent techniques. Singh et al., [14] developed and successfully implemented a pattern recognition approach to reactive navigation based on real time sensory information. A heuristic fuzzy neuro network is developed for pattern-mapping between quantized ultrasonic sensory data and the velocity commands to the robot. The design goal was to enable an autonomous mobile robot to navigate safely and efficiently to target position in a previously unknown environment. To build the desired mapping between perception and motion, usefully heuristic rules were combined with the fuzzy kohonen clustering network (FKCN).

In Yung and Ye [15] paper, an alternative training approach to the EEM based training method is presented and fuzzy reactive navigation architecture is described. Using the rule base learned from the new method, the proposed fuzzy reactive navigator fuses the obstacle avoidance behavior and goal seeking behavior to determine its control actions The new training method is 270 times faster in learning speed; and is only $4 \%$ of the learning cost of the EEM method, where adaptability is achieved with the aid of an environment evaluator. Nakrania and Joshid [16] discussed the control of autonomous intelligent robotic agent operating in unstructured changing environments. Online learning as a useful method producing intelligent machines for inaccessible environments was introduced. In these environments it is required to perform online learning through interaction with the real environment and performing any adaptation within short time intervals. Under such conditions, robotic agents have to be adaptive. Cao et al., [17] describe a neuro-fuzzy control method for the navigation of an AGV robot. An overall system design and development was presented. The neuro-fuzzy computation and its application for mobile robot navigation were discussed. The system that was to be controlled is an electrically propelled mobile vehicle named Bearcat II, which is a computer controlled intelligent system. As autonomous navigation requires a number of heterogeneous capabilities, including the ability to execute elementary goal-achieving actions, like reaching a given location; to reach in real time to unexpected events, like the sudden appearance of an obstacle; to determine the robot's position; and to adapt to changes in the environment, the paper introduces a neuro-fuzzy control method for navigation of an autonomous guided vehicle (AGV) robot.

Carpenter et al., [18] introduce a neural integrated fuzzy controller (NiF-T), which integrates the fuzzy logic representation of human knowledge with the learning capability of neural networks, is developed for nonlinear dynamic control problems. It covers integrated sensing, control, actuator modules, real-time performance, ability to successfully handle noisy sensor signals, reactive controller design which captures high-level, linguistically based human expertise in a set of fuzzy rules, training of neural networks directly with fuzzy rules instead of numerical sample data, as well as learning capabilities and general applicability. NiF-T architecture comprises "of three distinct parts: 1) fuzzy logic membership functions (FMF), 2) a rule neural network (RNN), and 3) and output-refinement neural network (ORNN). FMF are utilized to fuzzify sensory inputs. RNN interpolates the fuzzy rule set; after defuzzification, the output is used to train ORNN. The weights of the ORNN can be adjusted on-line to fine-tune the controller". Only five rules were used to train the wall following behavior, while nine were used for the hall centering. Also, a robot convoying behavior was realized with only nine rules. For all of the described behaviors-wall following, hall centering, and convoying, their RNN's were trained only for a few hundred iterations and so are their ORNN's trained for only less than one hundred iterations to learn their parent rule sets.

\section{RESEARCH METHOD}

The mobile robot is required to explore several paths in a maze, of a pattern of successive combinations of left and right turns. Its task is to reach a desired position at the end of one channel. 
The mobile robot uses a kind process, sequentially adopting cyclic pattern of the left and right turns [19]. Eventually, it ends up with the desired position, at which time a signal is injected, causing the robot to record the correct pattern. The mobile robot is assumed to be equipped with three physical ultrasonic sensors and one virtual sensor as shown in Figure 1.

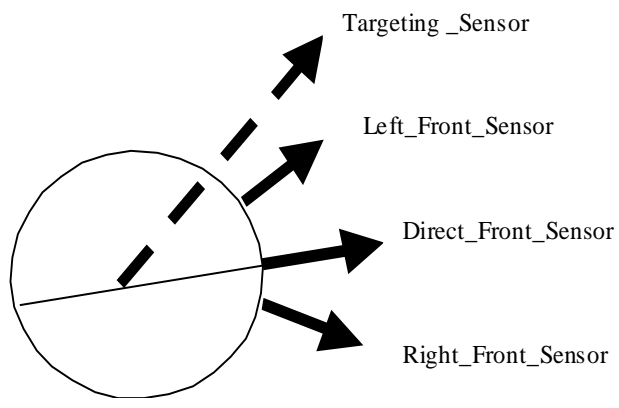

Figure 1. Mobile robot with frontal sensors

The physical sensors are used to detect obstacles in front of the robot, the right side, and the left side, respectively. The maximum distance that can be sensed by these sensors is assumed to be 8 meters [20]. The virtual sensor is used to guide the robot towards the target. This sensor is especially needed when the target direction of movement is totally blocked by an obstacle [21]. The virtual sensor will guide the robot back towards the target once the obstacle is avoided.

Henceforth, the robot travels quickly and accurately along the track to accomplish any job that has been assigned. It is assumed that the robot will not face any traps (or get into a situation where it is required to backtrack or turn around). Such a problem is out of this paper scope. The four sensors provide the path planning system (in our case fuzzy logic system) of the robot with three distances front (dc), right (dr), left (dl), and target orientation (theta), respectively [22]. From these inputs, the fuzzy logic controller will make up a decision in which direction should the robot move in order to reach the target [23]. The neuro-fuzzy controller should pass through three stages, i.e., fuzzification, inference, and defuzzification as shown in Figure 2.

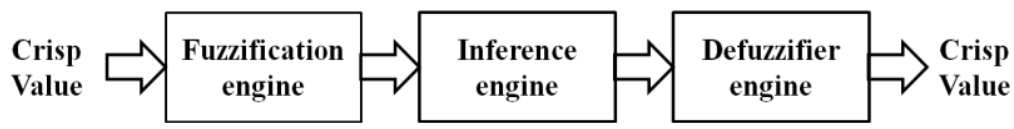

Figure 2. Neuro-fuzzy controller stages

\subsection{Fourty rules fuzzy navigator system}

The fuzzy logic controller (FLC40) was analyzed and tested for different cases based on the same parameters and rules used by $\mathrm{Xu}$ and Tso [24]. The robot motion results have been considered with relation to different cases. Problems were recorded and investigated and the reasons behind the failure of this robot, in these cases, were related to the limited number of the sets used (FAR, NEAR), and the limited angle of orientation (turning angle), which are five sets. Due to this limitation, the robot touches the obstacles slightly in all cases considered as shown in Figure 3. To avoid these problems, a relaxation of the rules was done by increasing the number of sets for the input distances from two to five sets; accordingly, the number of rules was increased to 625 activation rules which will be discussed in the next section.



Figure 3. FLC40, simulated motion 


\subsection{Fuzzy navigator system of 625 rules}

As it has been already noted, the FLC40 is not capable to avoid collision with the edges of the obstacles in all cases. The main reason behind that failure is the low resolution due to two fuzzy sets, i.e., FAR and NEAR. An improvement to the system can be easily made by increasing the number of fuzzy sets in order to achieve better resolution. In this paper, it is proposed to increase the fuzzy sets to five linguistic labels (VL, L, M, S, VS) as shown in Figure 4(a-c). The fuzzy sets in this case become shorter than before, so the accuracy and the performance of the controller are improved. As the number of sets is increased the fuzzy rules are increased as well up to 625 activation rules $(5 \times 5 \times 5 \times 5=625$ activation rules $)$.

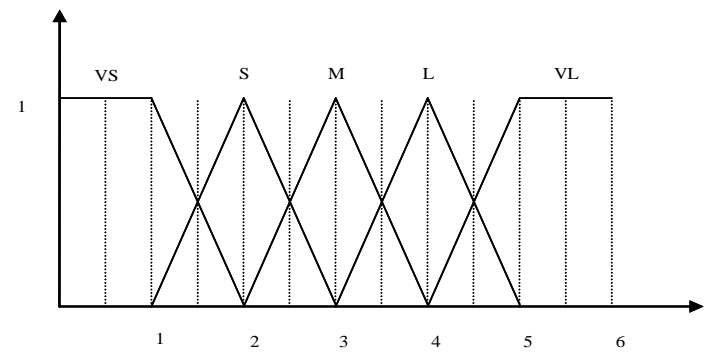

(a) VL: Very Large, L: Lange, M: Medium, S: Small, VS: Very Small

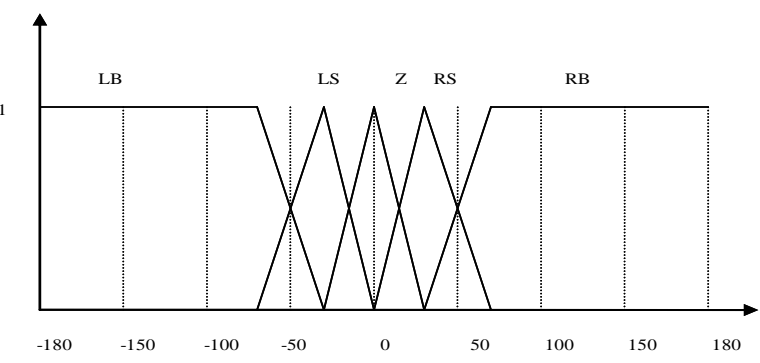

(b) LB: target_Left Big, LS: target_Left Small, Z: target_Zero, RS: targer_Right Small, RB: target_Right Big

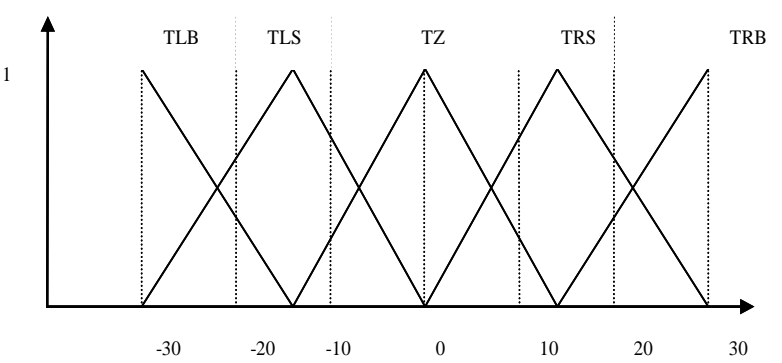

(c) TLB: Turn_Left_Big, TLS: Turn_Left_Small, TZ: Turn_Zero, TRS: Turn_Right_Small, TRB: Turn_Right_Big

Figure 4. (a) Distance, (b) Target orientation, (c) Turning angle membership function

As an example, a sample is presented where the activation rules are:

IF $d r$ is $L$ and dc is $L$ and $d l$ is $L$ and tr is LB THEN Sa is TLB

IF $d r$ is $S$ and $d c$ is $M$ and $d l$ is $L$ and tr is RB THEN Sa is TZ

IF $d r$ is $L$ and $d c$ is $M$ and $d l$ is $S$ and tr is RS THEN Sa is TRS

The results obtained from this improved fuzzy logic controller have been improved. The robot avoids collision with the obstacles as shown in Figure 5, but the main problem in using that improved controller is the processing time. It is very long, since the number of rules is high and requires more time to create a decision and this will affect the response time of the robot.



Figure 5. Improvement FLC625 success cases 


\subsection{Development of the improved fuzzy navigator system}

As it has been already noted that the FLC40 is not capable to avoid collision with the edges of the obstacles in all cases, also, FLC625 can avoid all the obstacles but unfortunately with low response since the processing time is large. An improvement to the system can be easily made by slightly increasing the number of fuzzy sets in order to achieve better resolution. In this paper, it is proposed to increase the fuzzy sets to three linguistic labels (L, M, S) as shown in Figure 6(a-c). The fuzzy sets in this case become shorter than before, so the accuracy and the performance of the controller are improved. As the number of sets is increased the fuzzy rules are increased as well up to 135 activation rules $(3 \times 3 \times 3 \times 5=135$ activation rules $)$.

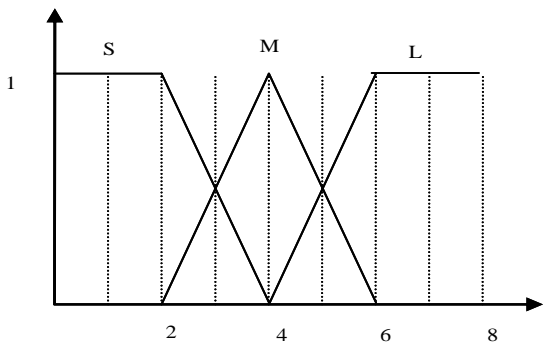

(a) L: Large, M: Medium, S: Small

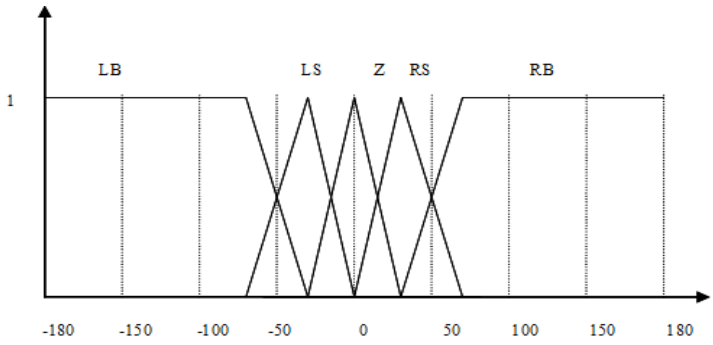

(b) LB: target_Left Big, LS: target_Left Small, Z: target_Zero, RS: targer_Right Small, RB: target_Right Big

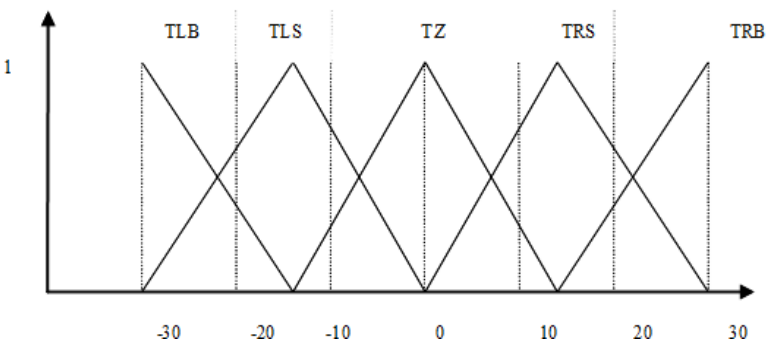

(c) TLB: Turn_Left_Big, TLS: Turn_Left_Small, TZ: Turn_Zero, TRS: Turn_Right_Small, TRB: Turn_Right_Big

Figure 6. (a) Distance, (b) Target orientation, (c) Turning angle membership function

As an example, a sample is presented where the activation rules are:

IF $d r$ is $\mathrm{L}$ and dc is $\mathrm{L}$ and $\mathrm{dl}$ is $\mathrm{L}$ and tr is LB THEN Sa is TLB

IF $\mathrm{dr}$ is $\mathrm{S}$ and $\mathrm{dc}$ is $\mathrm{M}$ and $\mathrm{dl}$ is $\mathrm{L}$ and tr is RB THEN Sa is TZ

IF $d r$ is $L$ and $d c$ is $M$ and $d l$ is $S$ and tr is RS THEN Sa is TRS

The results obtained from this improved fuzzy logic controller have been improved. The robot avoids collision with the obstacles as shown in Figure 7. Also, the processing time for this small number of rules is very short which increased the response time of the robot comparing with using 625 activation rules as in reference [24]. Furthermore, this paper focused on evaluating the performance of this fuzzy controller by comparing the results with other approaches such as $\mathrm{Xu}$ and Teso [24] and Ayman [25]. These comparisons are presented in the next section.

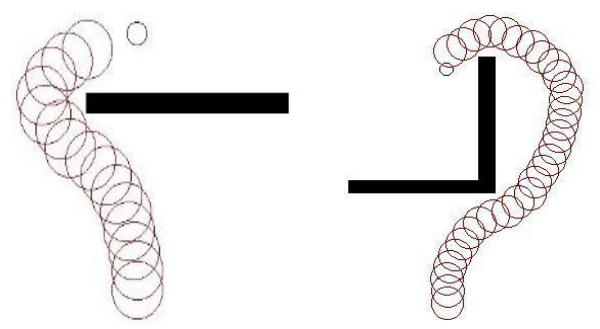

Figure 7. Improvement FLC135 success cases 


\section{RESULTS AND ANALYSIS}

The main problem in the fuzzy logic controller presented in reference [24] is the inference block, which consists of a large number of rules that need a long processing time. To solve this problem of processing time, the inference engine was optimized by using three sets for each sensor instead of five sets and keep the target orientation as five sets as in [24]. Therefore, the numbers of rules are reduced to $79 \%$ and the performance for mobile robot still same as in FLC625 activation rules [24] as shown in Figure 8.

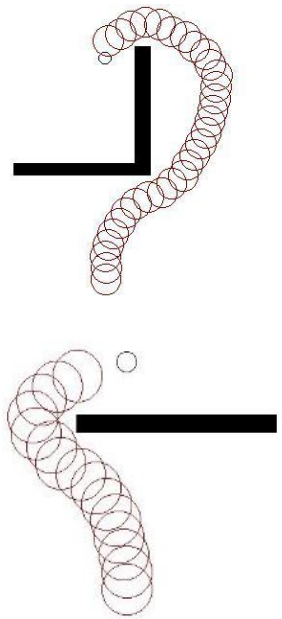

(a)

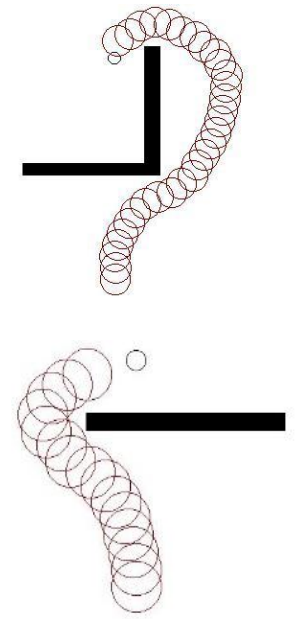

(b)

Figure 8. (a) FLC625 cases, (b) FLC135 cases

The main advantage gained by utilizing the optimized fuzzy controller that reduce number of rules in turn reduce the inference time from $1736 \mu \mathrm{S}$ to $349 \mu \mathrm{S}$ which increases the response of the controller and improves the performance of the robot. Practically, simulation-using PC doesn't show the differences in the CPU time for the three controllers since the PC is very fast and the response of the hardware is slow [26]. The CPU time for the three controllers is noticed when using micro controller chip to control the robot motion and download the program to the implemented robot. In the FLC40, the controller response time will be faster than both controllers, but the performance is limited. On the other hand, the FLC625 worked out well but with low response, which introduced a deficiency in the robot motion (create a dead point in the robot controller). The FLC135 increased the response of the whole controller and improves the performance of the robot motion. All these results are summarized in Table 1.

Table 1. The performance evaluation of FLC40, FLC625, and FLC135

\begin{tabular}{|c|c|c|c|c|}
\hline \multirow[t]{2}{*}{ Variable } & \multirow[t]{2}{*}{ Performance } & \multicolumn{3}{|l|}{ CPU Time $(\mu \mathrm{S})$} \\
\hline & & Fuzzification time & Inference time & Total CPU time \\
\hline FLC 40 & Slightly or severely colliding with the obstacles & $527 \mu \mathrm{S}$ & $132 \mu \mathrm{S}$ & $659 \mu \mathrm{S}$ \\
\hline FLC 625 & $\begin{array}{l}\text { Avoid collision with the obstacles and } \\
\text { smoothly reaches the target }\end{array}$ & $1038 \mu \mathrm{S}$ & $1736 \mu \mathrm{S}$ & $2757 \mu \mathrm{S}$ \\
\hline FLC 135 & $\begin{array}{l}\text { No collision at all with the obstacles with high } \\
\text { response time }\end{array}$ & $840 \mu \mathrm{S}$ & $349 \mu \mathrm{S}$ & $1189 \mu \mathrm{S}$ \\
\hline
\end{tabular}

\section{CONCLUSION}

The performance of the FLC625 algorithm is good and slightly improved the performance of the robot compared to the FLC40 since the robot doesn't touch any obstacle and the robot avoids collision with any obstacles as shown in the above cases. But the inference time is much more than the FLC40. However, the proposed approach that design on optimizing number of sets in the fuzzy logic controller reduced the processing time and increased the performance. The response of the implemented robot has shown an excellent reduction with respect to the response times. 


\section{ACKNOWLEDGEMENTS}

The author is grateful to the Applied Science Private University, Amman, Jordan, for the full financial.

\section{REFERENCES}

[1] E. Aguirre and A. González, "Fuzzy behaviors for mobile robot navigation: design, coordination and fusion," International Journal of Approximate Reasoning, vol. 25, no. 3, pp. 255-289, 2000.

[2] S. K. Harisha, P. Kumar, M. Krishna, and S. C. Sharma, "Fuzzy logic reasoning to control mobile robot on predefined strip path," World Academy of Science, Engineering and Technology, vol. 18, pp. 642-646, 2008.

[3] A. Saffiotti. S. Pradhan, D. Parhi, and A. Panda, "Fuzzy logic techniques for navigation of several mobile robots," Applied Soft Computing, vol. 9, no. 1, pp. 290-304, 2009.

[4] S. Parasuraman, B. Shirinzadeh, and V. Ganapathy, "Mobile robot navigation using alpha level fuzzy logic system: experimental investigations," 2008 IEEE International Conference on Systems, Man and Cybernetics, Singapore, pp. 1878-1884, 2008.

[5] T. J. Ross, "Fuzzy logic in engineering applications," Mc Graw-Hill Inc, New York, 1998.

[6] L. A. Zadeh, "The roles of fuzzy logic and soft computing in the conception, design and deployment of intelligent systems," Software Agent and Soft Computing Towards Enhancing Machine Intelligence, pp. 181-190, 1997.

[7] K. Tanaka, "An introduction to fuzzy logic for practical application," 1st Edition, Spring-Verlag, New York, 1996.

[8] R. Callan, "The essence of neural networks," Prentice-Hall, Europe, 1999.

[9] U. Orozco-Rosas, O. Montiel, and R. Sepúlveda, "Mobile robot path planning using membrane evolutionary artificial potential field," Applied Soft Computing, vol. 77, pp. 236-251, 2019

[10] L. Fausett, "Fundamentals of neural networks," Prentice-Hall, Europe, 1994

[11] A. R. Medsker, "Hybrid intelligent systems," Kluwer Academic Publishers, Europe, 1995

[12] A. Kumar and V. Kumar, "Performance analysis of optimal hybrid novel interval type-2 fractional order fuzzy logic controllers for fractional order systems," Expert Systems with Applications, vol. 93, no. 1, pp. 435-455, 2018.

[13] M. Zabihi and A. Alasty, "Dynamic stability and control of a novel handspringing robot," Mechanism and Machine Theory, vol. 137, pp. 154-171, 2019.

[14] M. K. Singh, D. R. Parhi, S. Bhowmik, and S. K. Kashyap, "Intelligent controller for mobile robot: Fuzzy logic approach," The 12 International Conference of International Association for Computer Methods and Advances in Geomechanics (IACMAG), pp. 1755-1762, 2008.

[15] N. H. C. Yung and Ye C., "An intelligent mobile vehicle navigator based on fuzzy logic and reinforcement learning," IEEE Transactions on Systems, Man, and Cybernetics, Part B (Cybernetics), vol. 29, no. 2, pp. 314-321, April 1999.

[16] N. Nakrania and M. Joshib, "An intelligent fuzzy based hybrid approach for parallel parking in dynamic environment," Procedia Computer Science, vol. 133, pp. 82-91, 2018.

[17] J. Cao, X. Liao, and E. L. Hall, "Reactive navigation for autonomous guided vehicle using the neuro-fuzzy techniques," Proceedings of SPIE-The International Society for Optical Engineering, vol. 3837, pp. 108-117, 1999.

[18] G. A. Carpenter, S. Grossberg, N. Markuzon, J. H. Reynold, and D. B. Rosen, "Fuzzy ARTMAP: A neural network architecture for incremental supervised learning of analogue multi-dimensional maps," IEEE Transactions on Neural Networks, vol. 3, no. 5, pp. 698-713, Sept. 1992.

[19] Z. Li, C. Yang, C. Su, J. Deng, and W. Zhang, "Vision-based model predictive control for steering of a nonholonomic mobile robot," IEEE Transactions on Control Systems Technology, vol. 24, no. 2, pp. 553-564, March 2016.

[20] M. A. Kamel, X. Yu, and Y. Zhang, "Fault-tolerant cooperative control design of multiple wheeled mobile robots," IEEE Transactions on Control Systems Technology, vol. 26, no. 2, pp. 756-764, March 2018.

[21] W. Ye, Z. Li, C. Yang, J. Sun, C. Su, and R. Lu, "Vision-based human tracking control of a wheeled inverted pendulum robot," IEEE Transactions on Cybernetics, vol. 46, no. 11, pp. 2423-2434, Nov. 2016.

[22] J. Huang, N. Zhou, and M. Cao, "Adaptive fuzzy behavioral control of second-order autonomous agents with prioritized missions: theory and experiments," IEEE Transactions on Industrial Electronics, vol. 66, no. 12, pp. 9612-9622, Dec. 2019.

[23] J. Huang, N. Zhou, and M. Cao, "Adaptive fuzzy behavioral control of second-order autonomous agents with prioritized missions: theory and experiments," IEEE Transactions on Industrial Electronics, vol. 66, no. 12, pp. 9612-9622, Dec. 2019

[24] W. L. Xu and S. K. Tso," Real time self-reaction of a mobile robot in unstructured environment using fuzzy reasoning," Engineering Application of Artificial Intelligent, vol. 9, no. 5, pp. 475-485, 1996.

[25] A. A. AbuBaker, "An intelligent mobile robot controller using neuro-fuzzy techniques," Master Thesis, University of Jordan, 2002.

[26] J. Yu, J. Liu, Z. Wu, and H. Fang, "Depth control of a bioinspired robotic dolphin based on sliding-mode fuzzy control method," IEEE Transactions on Industrial Electronics, vol. 65, no. 3, pp. 2429-2438, March 2018. 